\title{
Congenital Distal Tibiofibular Synostosis - A Case Report
}

\author{
Sreenivas $\mathbf{T}^{1}$ \\ ${ }^{1}$ Department of Orthopedics, Kannur Medical College, PO Anjarakandy, Kannur, Kerala, India
}

\begin{abstract}
Congenital tibiofibular synostosis is the fusion of tibia and fibula since birth. So far there are many reports of congenital proximal tibiofibular synostosis in English literature, but congenital distal tibio fibular synostosis is very rarely described. Imaging studies by means of X rays, CT and MRI are required to rule out osteochondromas arising from distal tibia, fibula and other conditions. If the patient is symptomatic by means of deformity surgical intervention in the form of corrective osteotomy may be considered to prevent alternation of joint biomechanics. We report a rare case of congenital distal tibiofibular synostosis in a 21 year old female presented with complaints of deformity and pain on and off in left lower leg since childhood.
\end{abstract}

Key Words: Congenital; Synostosis; Deformity; Osteochondroma

\section{CASE REPORT}

A 21 year old female presented to the Orthopedic outpatient department with history of increasing deformity of her left lower leg and pain on and off since childhood. There was no history of trauma, infection or any surgical intervention. Pain in the form of vague discomfort was present all over the left leg but does not confined to the region of deformity. Written informed consent was obtained from the patient for the purpose of publication.

Clinical examination revealed abnormal prominence on postero-lateral aspect of distal fibula with 15 degrees of varus deformity of the distal tibia. There was no limb length discrepancy and no localized tenderness. Range of movements of the ipsilateral knee $\&$ ankle were painless and comparable to the normal side. There was no distal neurovascular deficit. On general physical examination there was no obvious swelling or deformities in other parts of the body.

Plain radiographs of left leg and ankle (both antero-posterior \& lateral) [Figure 1] demonstrated complete bony fusion of distal tibia and fibula at metadiaphyseal area and it was confirmed on computed tomography [Figure $2 \mathrm{a} \& 2 \mathrm{~b}$ ]. Radiological investigations didn't reveal any degenerative changes of the ankle joint. Routine blood investigations didn't show any abnormalities. Deformity correction was planned but patient refused the surgery. Symptomatic improvement in pain was noticed with analgesics.

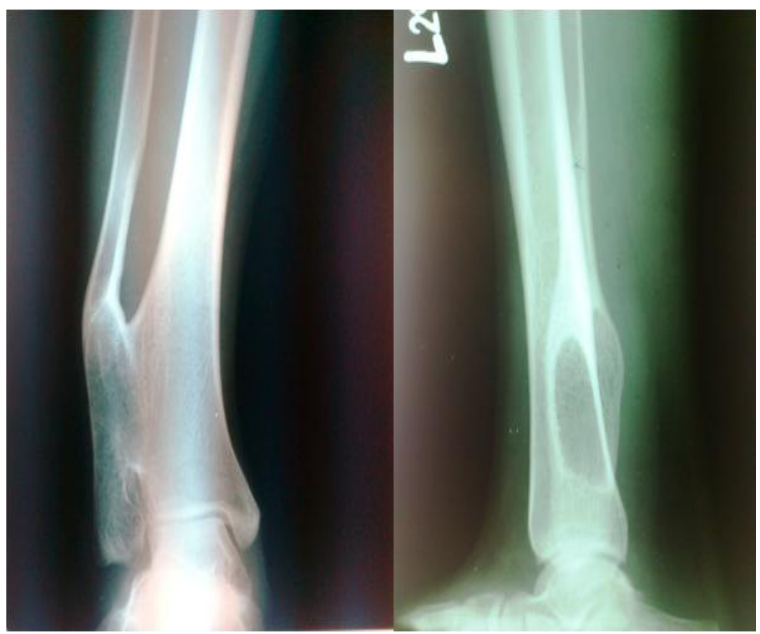

Figure 1: Anteroposterior and lateral Radiographs of left leg with ankle showing distal tibiofibular synostosis with varus at lower leg 


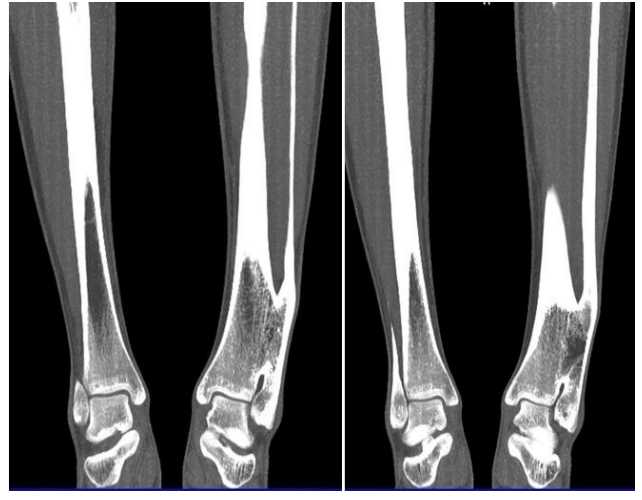

a.

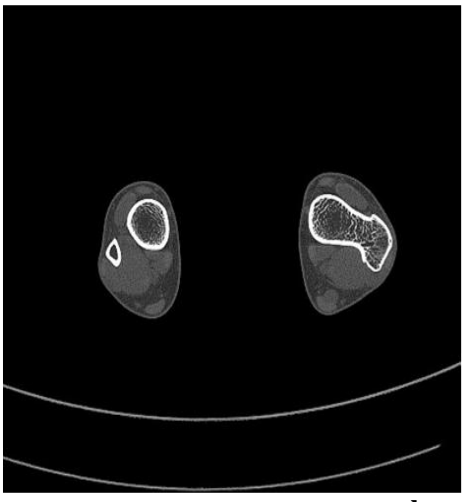

b.

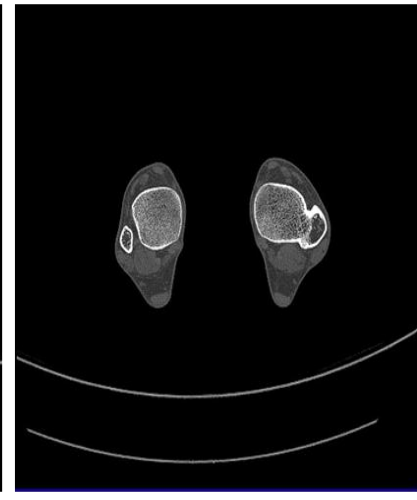

Figure 2: Computed tomography of both the legs showing normal tibia and fibula on right leg and distal tibiofibular synostosis on left side in both coronal (a) and axial sections (b)

\section{DISCUSSION}

Tibiofibular synostosis is very rarely described in literature. It encompasses two entities- proximal \& distal tibiofibular synostosis, the former is usually congenital whereas the later being acquired. Trauma is the most common cause of distal tibiofibular synostosis [1]. However it may also occur due to osteochondromas, fluorosis, fibro dysplasia ossificans progressiva, subperiosteal hemorrhages as in hemophilia or may be iatrogenic. Reports of isolated congenital distal tibiofibular synostosis are very few [2, 3, 4]. Hypothesis described in favour of congenital origin are intra-uterine trauma, infection, developmental arrest [1].

Fractures around the ankle [5] and the fracture at the same level can lead to distal tibiofibular synostosis due to disruption of interosseous membrane $[5,6]$ or it may follow an open reduction and internal fixation for an ankle fracture [7]. Pre-operative imaging by means of $\mathrm{x}$ rays, CT and MRI are useful to rule out any neoplastic or other conditions. On imaging, synostosis appears as a bony bridge connecting the two bones with or without deformity. Imaging has a preoperative role in excluding the underlying neoplastic and non-neoplastic conditions like hereditary causes (hemophilia) or metabolic conditions (scurvy and fluorosis) [1]. Neoplastic conditions like osteochondroma and ossifying parosteal Osteosarcoma can be excluded $[8,9]$. The varus deformity seen may be due variations in growth of distal tibia and fibula.
Since our patient had no history of trauma, nor undergone any surgical procedure $\&$ the presence of progressive deformity since childhood suggests that the origin of synostosis is most likely congenital. As the patient already has attained skeletal maturity, the chance of further increase in the deformity is less in our case and it is also true in the case of osteochondroma causing deformity. The pain in the leg might be due to soft tissue stretching around the ankle \& lower leg. If the deformity is not treated properly it might lead to early development of osteoarthritis in hip, knee \& ankle joints due to altered joint biomechanics.

\section{Acknowledgement: None}

\section{Conflict of Interest: None}

\section{Source of Funding: None}

\section{REFERENCES}

1. Jiang-Hue $\mathrm{Fu}$, Chyi-Chyuan Hwang, and Tai-Hung Chao. Tibiofibular synostosis in a military soldier. J Med Sci 2003;23(2):135138

2. O'Dwyer KJ. Proximal tibio-fibular synostosis. A rare congenital anomaly. Acta Orthop Belg. 1991;57(2):204-8

3. Grobelski M. Congenital tibiofibular synostosis of the distal end of the lower leg. Arch Orthop Unfallchir 1965;5; 57: 190-3

4. Jyoti Sureka, Ravi Kanth Jakkani, Munawwar Ahmed. Congenital distal tibiofibular synostosis. Radiology Case 
Reports 2012; 7(2). DOI: 10.2484/ rcr.v7i2.555

5. Munjal K, Kishan S, Sabharwal S. Posttraumatic pediatric distal tibiofibular synostosis: a case report. Foot Ankle Int 2004; 25(6):429-33

6. Vitale TD, Fallat LM. Distal tibiofibular synostosis and late sequelae of an ankle sprain. J Foot Surg 1990;29(1):33-6

7. Lee JY, Nam KY, Song KC. Distal tibiofibular synostosis after open reduction and internal fixation in a military soldier (A case report). Korean Foot Ankle Soc 2010;14(1):105-107

8. Bozkurt M, Doğan M, Turanli S. Osteochondroma leading to proximal tibiofibular synostosis as a cause of persistent ankle pain and lateral knee pain: a case report. Knee Surg Sports Traumatol Arthrosc 2004; 12(2):152-4. Epub 2003 Aug 27

9. Bessler W, Eich G, Stuckmann G, Zollikofer C. Kissing osteochondromata leading to synostoses. Eur Radiol 1997;7(4):480-5

How to cite this article: Sreenivas T. Congenital distal tibiofibular synostosis - a case report. Int $J$ Health Sci Res. 2021; 11(9): 178-180. DOI: https://doi.org/10.52403/ijhsr.20210927 